\title{
Factors Associated with Short-Term Hospital Readmission Rates for Breast Cancer Patients in Western Australia: An Observational Study
}

Jeffrey K Lai, PHB, Michael A Martin, BSc, PhD, Ramona Meyricke, BSc, Terry O’Neill, BSc, MS, PhD, Steven Roberts, BEC, MS, PhD

BACKGROUND: Unplanned hospital readmissions after surgical treatment for breast cancer are an indicator of morbidity. We explore the relationship between the rate of unplanned hospital readmissions within 42 days of initial treatment and various factors, including tumor size and histology, lymph node involvement, type of surgical treatment, mastectomy, or breast-conserving surgery, and patient demographics.

METHODS: $\quad$ Linked Western Australian cancer mortality and hospital morbidity data were used in the assessment of readmissions within a period of 42 days after initial surgical treatment for breast cancer. Planned admissions for adjuvant treatment such as chemotherapy or radiotherapy were deleted. Survival models for multiple events per subject were applied to analyze the data.

RESULTS: $\quad$ The analysis reveals that patients more likely to experience lower recurrence of short-term unplanned hospital readmissions include those with smaller tumors, private insurance, and who reside in metropolitan areas. The model also includes important two-way interaction terms involving tumor histology, area of residence, and surgical treatment, and between lymph node involvement and patient age.

CONCLUSIONS: This study suggests that the choice of breast-conserving surgery as a treatment for breast cancer does not invariably result in better postoperative morbidity, but rather, that other factors, including tumor size and patient demographics, play a critical role in the short-term. These results differ from a previous study of longterm hospital readmissions - country of birth and method of payment were found to be associated with short-term hospital admission but not with longterm readmissions. (J Am Coll Surg 2007;xx:xxx. (C) 2007 by the American College of Surgeons)

Breast cancer is a disease that affects about one-tenth of Australian women. Because of its devastating impact on the community, much research has been conducted on multiple aspects of the condition, including possible causative factors, methods of treatment and patient care, and preventative measures, such as breast screening. A number of clinical trials have concluded that breast cancer patients treated with breast-conserving surgery (BCS) have longterm survival rates similar to those

\section{Competing Interests Declared: None.}

This work was partially supported by a College of Business and Economics, Australian National University, Faculty Internal Grant Scheme.

Received October 19, 2006; Accepted November 8, 2006.

From the School of Finance and Applied Statistics, Australian National University, Canberra, Australia.

Correspondence address: Michael A Martin, BSc, PhD, School of Finance and Applied Statistics, Australian National University, Canberra ACT, 0200, Australia.email: Michael.Martin@anu.edu.au treated with mastectomy. ${ }^{1-3}$ This evidence is supported by a recent pooled analysis of six major clinical trials comparing mastectomy and BCS, which concluded that the two treatments have comparable effects on mortality, even after longterm followup. ${ }^{4}$ Other population-based studies have also shown that patients receiving mastectomy and BCS have similar survival rates.,

Apart from survival rates associated with the breast cancer treatment options of BCS and mastectomy, another question of great importance for breast cancer patients is the quality of life or morbidity associated with BCS and mastectomy. For example, although BCS and mastectomy have been shown to have similar longterm survival rates, it might be the case that one form of treatment affords a better quality of life (ie, less complications or better self-image), which, if known to breast cancer patients facing a treatment choice, would play an 


\section{Abbreviations and Acronyms}

BCS = breast-conserving surgery

WA $\quad=$ Western Australia

WAHMD $=$ Western Australia Hospital Morbidity Database

integral part in their choice of treatment. Along these lines, a recent study showed substantially greater levels of psychological morbidity in women who had BCS compared with those who had mastectomy. ${ }^{7}$ Until recently, the issue of physical morbidity for breast cancer patients treated with BCS compared with those treated with mastectomy has received little attention in the literature.

A recent study explored the relationship between longterm unplanned hospital readmissions and various factors, including tumor size and histology; lymph node involvement; patient demographics; and type of surgical treatment, mastectomy or BCS. ${ }^{8}$ This study found that factors associated with lower rates of longterm unplanned hospital readmissions include smaller tumor size, metropolitan area of residence, and initial surgical treatment being BCS. The purpose of this article is to augment these findings by focusing on the short-term morbidity of patients after surgical treatment for breast cancer. This short-term analysis will allow insight into acute risks inherent from factors such as tumor size and histology, type of surgical treatment, and patient demographics, on the morbidity of patients, including postoperative complications. Our results suggest that different factors are associated with short-term readmission rates than longterm readmission rates.

The aim of this article is to determine the factors associated with unplanned readmissions to hospital within a 42-day period after surgical treatment for Western Australian female breast cancer patients diagnosed after January 1, 1995. Planned readmissions are usually for checkups or additional adjuvant therapy after an operation. Unplanned readmissions reflect a need for medical treatment outside the treatment protocol arranged between patient and physician. Unplanned admissions are an important indicator of patient morbidity and a proxy for quality of life. For example, a comparison of unplanned hospital admission rates of breast cancer patients treated with BCS and mastectomy, allowing for other disease and patient characteristics, can provide valuable information on which surgical treatment offers a better quality of life and enables cancer patients to make more informed decisions about their choice of treatment. In addition, knowledge of which demographic variables appear associated with increased readmission risk can assist in identifying patients at increased risk for posttreatment physical morbidity.

\section{METHODS}

Data for the study were sourced from linked administrative data obtained from the Western Australian (WA) Department of Health Record Linkage Unit. The dataset was extracted from the WA Linked Database, a dynamic system linking three core data sources: the WA Cancer Registry, the WA Hospital Morbidity Database (WAHMD), and the WA Death Register. The WAHMD contains comprehensive patient demographic, diagnosis, and procedure information for each hospital admission in any WA hospital. The dataset used consists of the linked hospital, death, and WA Cancer Registry records containing the diagnosis, subsequent admissions to hospital, and death (where applicable) of approximately 3,000 women diagnosed with cancer in WA between January 1, 1995 and December 31, 1999. Data before 1995 were omitted because of the small number of observations with recorded tumor size measurement before this time. The WA Linked Database is unique in the Australian context and is an extraordinarily rich and comprehensive resource. ${ }^{9}$

For this analysis, surgical treatment was defined as the first procedure after a diagnosis of breast cancer. Date of diagnosis is defined for this study as the time at which the subject is recorded on the WA Cancer Registry, as none of the databases linked by the WA unit specifies diagnosis date. Date of first surgical treatment for breast cancer linked to each diagnosis was recorded on the WAHMD, and is used in this study as the start time for each patient. Explanatory variables included in the analysis were the first surgical treatment received by the subject (mastectomy or BCS), tumor size (diameter, measured in millimeters), nodal status (a categorical variable with categories no nodal involvement, one to three nodes, and four or more nodes ${ }^{10,11}$ ), tumor histology (a categorical variable classifying the tumor as ductal, lobular, or other), age of subject in years, subject's area of residence (rural/remote or metropolitan), subject's country of birth (Australia/New Zealand or not), marital status, whether or not they were Aboriginal or Torres Strait Islander, and method of payment (public or private). Table 1 provides summary information for each of 
Table 1. Summary Statistics for Subject Characteristics $(n=2,703)$

\begin{tabular}{|c|c|c|}
\hline & $n$ & $\%$ \\
\hline \multicolumn{3}{|l|}{ Operation } \\
\hline Mastectomy & 970 & 35.9 \\
\hline Breast-conserving & 1,733 & 64.1 \\
\hline \multicolumn{3}{|l|}{ Aboriginal } \\
\hline Yes & 23 & 0.9 \\
\hline No & 2,680 & 99.1 \\
\hline \multicolumn{3}{|l|}{ Marital status } \\
\hline Married/de facto & 1,867 & 69.1 \\
\hline Otherwise & 836 & 30.9 \\
\hline \multicolumn{3}{|l|}{ Method of payment } \\
\hline Public, eligible for Medicare & 1,340 & 49.6 \\
\hline Private, not insured & 89 & 3.3 \\
\hline Private, insured & 1,234 & 45.7 \\
\hline Ineligible for assistance & 40 & 1.5 \\
\hline \multicolumn{3}{|l|}{ Country of birth } \\
\hline Australia & 1,651 & 61.1 \\
\hline Other & 1,052 & 38.9 \\
\hline \multicolumn{3}{|l|}{ Area of residence } \\
\hline Rural/remote & 585 & 21.6 \\
\hline Metropolitan & 2,218 & 78.4 \\
\hline \multicolumn{3}{|l|}{ Lymph node involvement } \\
\hline Node-negative & 1,565 & 57.9 \\
\hline Node-positive (1-3 nodes) & 700 & 25.9 \\
\hline Node-positive (4+ nodes) & 332 & 12.3 \\
\hline Missing & 106 & 3.9 \\
\hline \multicolumn{3}{|l|}{ Tumor histology } \\
\hline Ductal & 2,151 & 79.6 \\
\hline Lobular & 331 & 12.2 \\
\hline Other & 221 & 8.2 \\
\hline \multicolumn{3}{|l|}{ TNM status } \\
\hline T1 & 1,701 & 62.9 \\
\hline $\mathrm{T} 2$ & 899 & 33.3 \\
\hline T3 & 103 & 3.8 \\
\hline \multicolumn{3}{|l|}{ Survival } \\
\hline Survived & 2,478 & 91.7 \\
\hline Died & 225 & 8.3 \\
\hline \multicolumn{3}{|l|}{ Tumor size (diameter, $\mathrm{mm}$ ) } \\
\hline Minimum & 1 & \\
\hline $1^{\text {st }}$ quartile & 12 & \\
\hline Median & 18 & \\
\hline $3^{\text {rd }}$ quartile & 25 & \\
\hline Maximum & 190 & \\
\hline \multicolumn{3}{|l|}{ Subject age (y) } \\
\hline Minimum & 23 & \\
\hline $1^{\text {st }}$ quartile & 51 & \\
\hline Median & 59 & \\
\hline $3^{\text {rd }}$ quartile & 69 & \\
\hline Maximum & 98 & \\
\hline
\end{tabular}

For categorical variables, percentages of subjects in each category are presented. For continuous variables, five-number summaries are presented. these variables. The extracted data comprised information from 2,703 patients. Of the 2,703 patients in the study, 106 did not have information on the nodal status variable described here. Of these 106 patients, the majority $(\mathrm{n}=103)$ had large tumors $(>50 \mathrm{~mm})$, and so results of our modeling are restricted to tumors within $50 \mathrm{~mm}$ in diameter, a value of clinical importance, as it reflects generally accepted clinical guidelines relating to tumor sizes beyond which breast-conserving therapy is contraindicated.

A program was written using SAS software (SAS Institute), which extracted the dates of all unplanned hospital readmissions from the WAHMD. Planned readmissions fail to consistently reflect morbidity, as it is common for patients with breast cancer to have multiple planned readmissions for adjuvant therapy, such as chemotherapy or radiotherapy, after initial operation. Crude rates of readmission are not useful because such planned readmissions account for a large proportion of readmissions. Before analysis, we removed planned admissions from the extracted dataset. We identified planned admissions by scanning the separation record on the WAHMD for procedure codes (from ICD-9 and ICD-10) that represent surgical aftercare procedures, such as chemotherapy, radiotherapy, occupational or physiotherapy, or procedure codes that represent a checkup or followup procedure. These codes were identified using both ICD-9 and ICD-10 codes. Any separation that included one or more of these codes was classified as a planned readmission and was removed from the dataset. Although we have simply used shortterm unplanned hospital readmissions as a proxy for short-term physical morbidity, there are potentially better, more specific, short-term morbidity indicators that might be considered. Such examples include breast and axillary wound infection after BCS or after mastectomy, seroma formation requiring aspiration, restricted shoulder range of motion requiring physical therapy or rehabilitation, breast or arm edema requiring treatment, margin reexcision after BCS, conversion from BCS to mastectomy, and complications of reconstruction after mastectomy. We elected to persist with the broader unplanned readmission indicator, as sufficiently specific ICD codes were unavailable for a substantial portion of patients in the study. We note that hospital readmission is more common in Australia than in the United States, where certain procedures are more commonly handled on an outpatient basis. 
Table 2. Distribution of Number of Unplanned Hospital Readmissions $(n=2,703)$

\begin{tabular}{|c|c|c|c|c|c|c|c|c|c|c|c|c|c|c|}
\hline \multirow[b]{3}{*}{ No. of readmissions } & \multirow{2}{*}{\multicolumn{2}{|c|}{ Overall }} & \multicolumn{4}{|c|}{ T1 } & \multicolumn{4}{|c|}{ T2 } & \multicolumn{4}{|c|}{ T3 } \\
\hline & & & \multicolumn{2}{|c|}{ M } & \multicolumn{2}{|c|}{ BCS } & \multicolumn{2}{|c|}{$\mathbf{M}$} & \multicolumn{2}{|c|}{ BCS } & \multicolumn{2}{|c|}{ M } & \multicolumn{2}{|c|}{ BCS } \\
\hline & $\mathbf{n}$ & $\%$ & $\mathbf{n}$ & $\%$ & $\mathbf{n}$ & $\%$ & $\mathbf{n}$ & $\%$ & $\mathbf{n}$ & $\%$ & $n$ & $\%$ & $\mathrm{n}$ & $\%$ \\
\hline 0 & 2,036 & 75.3 & 371 & 87.7 & 908 & 71.1 & 423 & 88.7 & 261 & 61.8 & 64 & 91.4 & 9 & 27.3 \\
\hline$\overline{1}$ & 585 & 21.6 & 44 & 10.4 & 326 & 25.5 & 49 & 10.3 & 139 & 32.9 & 4 & 5.7 & 23 & 69.7 \\
\hline 2 & 71 & 2.6 & 8 & 1.9 & 39 & 3.1 & 4 & 0.8 & 18 & 4.3 & 1 & 1.4 & 1 & 3 \\
\hline 3 & 9 & 0.3 & 0 & 0 & 3 & 0.2 & 1 & 0.2 & 4 & 0.9 & 1 & 1.4 & 0 & 0 \\
\hline 4 & 1 & 0.0 & 0 & 0 & 1 & 0.1 & 0 & 0 & 0 & 0 & 0 & 0 & 0 & 0 \\
\hline 5 & 1 & 0.0 & 0 & 0 & 1 & 0.1 & 0 & 0 & 0 & 0 & 0 & 0 & 0 & 0 \\
\hline Total & 2,703 & & 423 & 25 & 1,278 & 75 & 477 & 53 & 422 & 47 & 70 & 68 & 33 & 32 \\
\hline
\end{tabular}

BCS, breast-conserving surgery; $\mathrm{M}$, mastectomy.

Number of readmissions was broken down further by TNM category and first surgical treatment for breast cancer. Percentages in the body of the table reflect percentages within each column. Percentages given in the "Total" row reflect proportions of each type of surgical treatment within the respective TNM size categories.

Our analysis of unplanned short-term hospital readmissions involves application of survival analysis methods to data with multiple or recurrent events per subject. This application uses an extension of the Cox proportional hazards model to allow for intrasubject correlation inherent in the data. The correlation derives from the fact that the probability of a given subject having a hospital readmission, and the timing of this readmission, is dependent on the number and timing of previous hospital readmissions for the subject. The model used in this article was proposed by Prentice and colleagues, ${ }^{12}$ and will be referred to as the PWP model. The PWP model was selected because, in addition to handling the intrasubject correlation inherent in the data, it successfully handles the ordered nature of the hospital readmission data, ie, the model allows for the fact that a subject cannot be at risk for their hospital readmission until they have experienced previous readmissions. The PWP model is described in detail by Therneau and Grambsch, ${ }^{13}$ where it is also referred to as the conditional model. The model is particularly suited to this type of data.

To implement the PWP model, each subject is represented as a series of observations with time intervals: $\left(0, T_{1}\right],\left(T_{1}, T_{2}\right], \cdots,\left(T_{N}, \min \left(D_{i}, C_{i}\right)\right]$, where $T_{i}$ is the time of the $i^{\text {th }}$ hospital readmission, $D_{i}$ is the time of death of subject $i$ in the event that subject $i$ dies, although $C_{i}$ is the time at which subject $i$ ceased to be under observation in the event that subject $i$ is censored, in the case of this study at 42 days. By including death as a competing event, the PWP model is able to produce a global assessment of treatment effect with respect to both hospital readmission and patient's mortality risk. The PWP model was implemented using the statistical package S-Plus (Version 6.1; Insightful Corporation,
2002, available at: http://www.insightful.com). For our application, the majority of subjects experienced two or fewer unplanned hospital readmissions within the predefined 42-day period from first treatment. Table 2 summarizes the distribution of number of readmissions for patients in our study, also breaking down the number of readmissions by type of surgical treatment and TNM status of the tumor. The dataset did not contain explicit information on tumor TNM status. Using information on tumor size, we classified each tumor as T1, T2, or T3. Our dataset did not specify if tumors had, for example, penetrated the breast wall, and so we were unable to classify tumors as T4.

\section{RESULTS}

An initial amalgamated PWP model, including all covariates and two-way interactions, was fitted to the data. Using this initial model, backward elimination was used to find a final model. Backward elimination is a stepwise model-selection technique that, at each step, removes the "least important" variable (the variable with the largest $\mathrm{p}$ value). The process terminates when the least important variable has a $\mathrm{p}$ value $<0.05$. In implementing this process, we adhered to the standard modeling convention that, if a variable was present in an interaction term, then the main effect for this variable should also be included in the model, irrespective of its individual $p$ value. Our initial implementation of backward elimination led to the conclusion that there was no differentiation between node negative and node positive (one to three nodes) for the purposes of modeling short-term readmission rates. For this reason, we collapsed node negative and node positive (one to three nodes) into a single category, "low number of involved nodes" and 
Table 3. Summary of Results from the Selected Prentice, William, and Peterson ${ }^{12}$ Model Fit to the Hospital Readmission Data

\begin{tabular}{|c|c|c|c|}
\hline Covariate & Coef & Exp(coef) & p Value \\
\hline Tumor size (effect per mm) & 0.0071 & 1.007 & 0.0005 \\
\hline \multicolumn{4}{|l|}{ Tumor histology } \\
\hline Ductal (baseline) & - & - & - \\
\hline Lobular & 0.01134 & 1.011 & 0.77 \\
\hline Other & 0.02379 & 1.024 & 0.44 \\
\hline \multicolumn{4}{|l|}{ Area of residence } \\
\hline Rural/remote (baseline) & - & - & - \\
\hline Metropolitan & -0.1291 & 0.879 & 0.00039 \\
\hline \multicolumn{4}{|l|}{ Lymph node } \\
\hline- or $+(1-3$ nodes $)$ (baseline) & - & - & - \\
\hline$+(4+$ nodes $)$ & -1.3346 & 0.263 & 0.0015 \\
\hline \multicolumn{4}{|l|}{ Country of birth } \\
\hline Australia/NZ (baseline) & - & - & - \\
\hline Outside Australia/NZ & -0.018 & 0.982 & 0.4 \\
\hline \multicolumn{4}{|l|}{ Method of payment } \\
\hline Public patient (baseline) & - & - & - \\
\hline Private patient & -0.0682 & 0.934 & 0.0011 \\
\hline Age & -0.0196 & 0.981 & 0.2 \\
\hline$(\mathrm{Age})^{2}$ & 0.0002 & 1 & 0.11 \\
\hline \multicolumn{4}{|l|}{ Surgical treatment } \\
\hline Mastectomy (baseline) & - & - & - \\
\hline BCS & 0.02062 & 1.021 & 0.56 \\
\hline \multicolumn{4}{|l|}{ Tumor histology } \\
\hline Area interaction (ductal/rural baseline) & - & - & - \\
\hline Area metro interaction (lobular/metro) & -0.02651 & 0.974 & 0.47 \\
\hline Area metro interaction (other/metro) & -0.07125 & 0.931 & 0.015 \\
\hline Surgical treatment interaction-ductal/mastectomy (baseline) & - & - & - \\
\hline Surgical treatment interaction-lobular/BCS & 0.0891 & 1.093 & 0.0046 \\
\hline Surgical treatment interaction - other/BCS & -0.0258 & 0.975 & 0.36 \\
\hline Age/age $^{2}$ nodal involvement interaction $(0-3$ nodes baseline $)$ & - & - & - \\
\hline Age-nodal involvement ( $4+$ nodes) interaction & 0.0477 & 1.049 & 0.0015 \\
\hline$(\text { Subject age })^{2}$ nodal involvement $(4+$ nodes) interaction & -0.00037 & 1 & 0.0041 \\
\hline Country of birth - surgical treatment interaction-Australian/mastectomy (baseline) & - & - & - \\
\hline Country of birth - surgical treatment interaction-outside Australia/BCS & -0.06935 & 0.933 & 0.001 \\
\hline
\end{tabular}

Coef, coefficient.

Tumor size is measured as diameter of tumor $(\mathrm{mm})$, area of residence was coded 0 for rural/remote and 1 for metropolitan, first surgical treatment was coded as 0 for mastectomy and 1 for breast-conserving surgery, lymph node status was a factor with two levels (ie, $0-3$ nodes and $4+$ nodes), and tumor histology was a factor with three levels (ie, ductal, lobular, and other).

repeated the backward elimination process. The PWP model makes a proportional hazards assumption within each event stratum, and a diagnostic check of this assumption using the Schoenfeld residuals from the chosen model fit revealed that the proportional hazard assumption was reasonable with respect to all variables.

Fitted model coefficients and associated $\mathrm{p}$ values for the final model fit are summarized in Table 3. Standard errors of coefficients (not reported) used in calculating $\mathrm{p}$ values were obtained using a robust jackknife approach, as outlined in Therneau and Grambsch. ${ }^{12}$ For brevity, stan- dard errors and $\mathrm{z}$ scores are not reported in Table 3. The chosen model contains both main effects and interactions, the combinations of which paint a rich but complex picture of how the relevant factors are associated with short-term unplanned hospital readmissions. The broad interpretations of our modeling are summarized in Table 4.

The model fit reveals that patients more likely to experience lower recurrence of short-term unplanned hospital readmissions include those with smaller tumors, with private insurance, and who reside in metropolitan areas. The model also includes substantial two-way in- 
Table 4. Interpretation of Final Prentice, William, and Peterson ${ }^{12}$ Model Fit (Effects of Significant Predictors as a Proportion of the Baseline Hazard of Readmission)

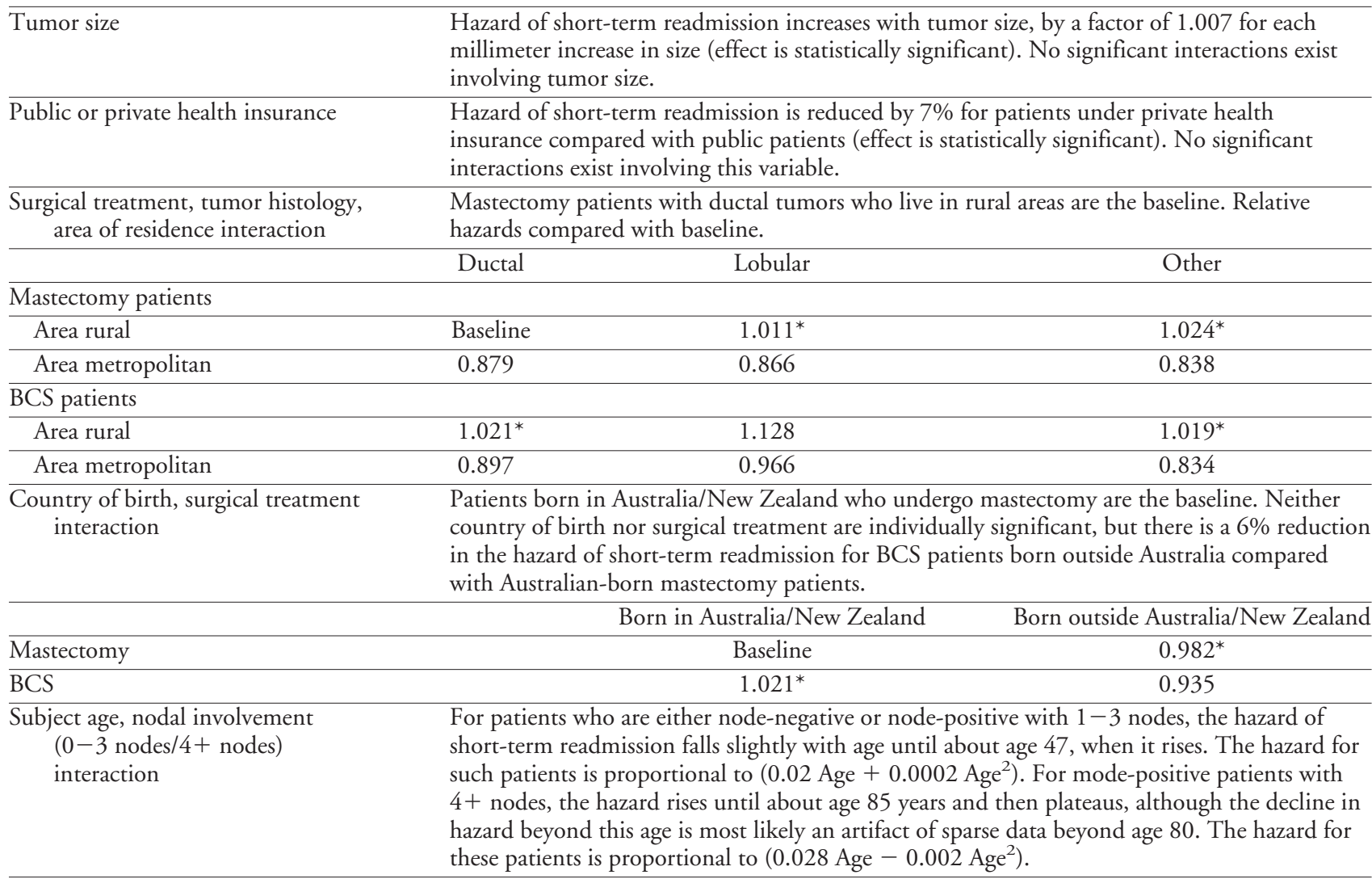

BCS, breast-conserving surgery.

*Statistically nonsignificant results against the baseline.

teraction terms involving tumor histology; area of residence; and surgical treatment, and between nodal involvement and patient age. Generally, metropolitanbased patients appear to have hazard rates $>10 \%$ lower than their rural/remote-based counterparts, but with slightly higher rates of short-term readmission for BCS patients with ductal or lobular tumors. For patients born outside Australia/New Zealand, the hazard of readmission is reduced by about $5 \%$ for those whose surgical treatment was BCS compared with mastectomy. Significant interactions also exist between subject age and nodal involvement. A cursory inspection of the model coefficients in Table 3 suggests that patients with four or more positive nodes experience substantially improved short-term readmission outcomes over patients with three or fewer positive nodes. Ehe effect of nodal involvement on short-term readmission rates needs to be interpreted particularly carefully because of the significant interaction between it and patient age, and the fact that, generally, high nodal involvement is correlated with larger tumors. Taking into account the interaction between nodal involvement and age (see Table 4), and noting that the median tumor size for the " 0 to 3 nodes" group is $16 \mathrm{~mm}$ compared with $25 \mathrm{~mm}$ for the " $4+$ nodes" group, the model predicts that patients with zero to three positive nodes aged between about 38 and 90 years experience a lower relative hazard rate than patients with more than four positive nodes in the same age group. For patients outside the 38 to 90 years age group, the model predicts the reverse-that patients in this age group with more than four positive nodes have a slightly lower relative hazard than those with zero to three positive nodes. This result is very likely an artifact of statistical modeling, as there are only two patients in the older than 90 years age group and the proportion of patients under 40 in the dataset is only $7 \%$ of total data.

Considering the interaction between country of birth and surgical treatment, the extent to which surgical treatment affects the hazard of readmission differs between patients born in Australia/New Zealand and those 
born outside Australia/New Zealand. For patients born in Australia/New Zealand, the hazard does not change substantially for patients whose treatment was mastectomy compared with those whose treatment was BCS. Although for patients born outside Australia/New Zealand, the hazard is about $5 \%$ lower for patients whose treatment was BCS compared with those whose treatment was mastectomy.

Several previous studies have considered morbidity associated with breast cancer treatments in several countries. Studies by Taylor ${ }^{14}$ and Shrank and colleagues ${ }^{15}$ consider morbidity associated with axillary lymph node dissection as part of breast cancer treatment, and conclude that the procedure has substantial, longterm morbidity consequences for patients. More generally, Hynes and colleagues ${ }^{16}$ showed that morbidity and readmission rates after treatment for breast cancer did not differ substantially between Veterans Administration hospitals and private-sector hospitals in the United States. Nevertheless, these studies did not focus on the issue of how a patient's choice of mastectomy or BCS impacted hospital readmission rates.

The importance of area of residence on unplanned short-term readmission experience is interesting and has obvious public health implications. After taking into account the interaction terms involving area of residence, subjects residing in metropolitan areas were still generally predicted to have substantially lower risk-by a factor of about $10 \%$ - of unplanned readmission than their rural/remote counterparts, perhaps reflecting that these patients have more initial treatment options available to them. It can be anticipated that rural patients are more likely to undergo mastectomy in cases where metropolitan patients in a similar situation might opt for BCS, notably in cases of small tumors. The interaction between area of residence and tumor histology is also of interest, with metropolitan patients with lobular tumors undergoing BCS appreciably worse off (relative hazard $97 \%$ of baseline) than corresponding patients undergoing mastectomy (relative hazard $87 \%$ of baseline).

Our previous results from the modeling of longterm hospital readmission experiences offers an interesting comparison with short-term results illustrated in this article. In both investigations, tumor size and area of residence are important indicators of readmission rates, but key differences emerge between the short- and longterm cases. For example, factors such as country of birth and method of payment do not appear to be associated with longterm readmission rates, but they do have an impact on short-term readmissions. Several plausible reasons exist as to why method of payment might affect short-term readmissions. For example, patients with private health insurance typically have a choice of surgeon and public patients might not, so the availability of such a choice can lead to better surgical outcomes and lower risk of short-term complications. Also, private patients tend to receive longer postoperative care within their initial hospital visit, and so might be less likely to experience acute postoperative complications. Private health insurance might allow for other modes of postoperative care, which reduce the chance of circumstances requiring hospital readmission in the short term. The result that BCS patients born outside Australia/New Zealand experience lower risk of short-term hospital readmission than patients born within Australia or mastectomy patients born outside Australia is a curious one, for which we can see no obvious explanation. This result does not persist for longterm readmission rates. Most notably, unlike in the longterm analysis, a key result of this study suggests that the choice of BCS as a treatment for breast cancer does not invariably result in better postoperative morbidity, but rather, other factors, including tumor size and patient demographics, play a critical role in the short term.

Despite the results of this analysis being quite complex, they raise interesting and provocative questions related to surgical treatments for breast cancer. The results of this article conclude that mastectomy offers improved short-term morbidity over BCS in certain cases, but longterm analysis suggests that BCS can tend to result in better longterm readmission rates, and patients must weigh these competing results. Of course, such treatment choices must also be made in the context of many factors other than posttreatment morbidity, and this study does not address these issues. Use of unplanned hospital readmission as a proxy for morbidity ignores other types of morbidity that might not be treated through hospital admission, such as psychological impacts of treatment, and so on.

The role of tumor size in affecting readmission rates is clear from our analysis-larger tumors tend to be associated with increased risk of short-term readmission regardless of which surgical treatment is used, although at larger tumor sizes (TNM status T3). The descriptive statistics presented in Table 2 suggest substantially poorer short-term readmission rates for BCS patients 
than for mastectomy patients in this group. Note that the overwhelming majority $(92 \%)$ of patients in the study had tumors classified as T1 or T2. Current clinical practice guidelines vary widely by country, but the $\mathrm{Na}$ tional Health and Medical Research Council in Australia suggests that tumor size of 3 to $4 \mathrm{~cm}$ is often regarded as a "practical limit" beyond which local excision incurs a high risk of recurrence ${ }^{17}$ (p 52). In the United States, the commonly quoted National Comprehensive Cancer Network's guidelines for breast cancer care ${ }^{18}$ do not stipulate an absolute tumor size above which BCS should not be used, but do note a relative contraindication to BCS requiring radiation therapy for tumors $>5 \mathrm{~cm}$ (category $2 \mathrm{~B}$ ) in diameter. The summaries presented in Table 2 show that for our data, the proportion of patients undergoing BCS varies markedly with the TNM category of the tumor, from about $75 \%$ for patients with tumors classified as T1, to about $50 \%$ for patients with tumors classified as T2, and down to about 30\% for patients with tumors classified as T3, noting again that the number of patients in our study in the latter, T3 category was very small (only about 100).

Limitations of the present study must also be acknowledged. First, our study is based on data collected over only a relatively short period of time, as data on all the covariates included in our study were unavailable over a longer period. Also, although the Western Australian database is unique in the Australian context in linking critical data from several sources, it still lacks information on certain variables (eg, tumor stage, size of tumor as a proportion of breast size, degree of differentiation of tumor) that could be usefully considered when modeling hospital readmission data. Other areas where additional research is warranted include whether increased readmission rates associated with mastectomy are for reconstructive surgery, complications arising from mastectomy, or other medical treatment required in the longterm. Despite these limitations, the conclusions of our analysis allow an assessment of the factors associated with differential short-term hospital readmission rates for Western Australian breast cancer patients.

\section{Author Contributions}

Study conception and design: Martin, Meyricke,

O’Neill, Roberts

Acquisition of data: Meyricke

Analysis and interpretation of data: Lai, Martin,

Meyricke, O’Neill, Roberts
Drafting of manuscript: Lai, Martin, Roberts

Critical revision: Lai, Martin, Meyricke, O’Neill, Roberts

\section{REFERENCES}

1. Veronesi U, Saccozzi R, Del Vecchio M, et al. Comparing radical mastectomy with quadrantectomy, axillary dissection, and radiotherapy in patients with small cancers of the breast. $\mathrm{N}$ Engl J Med 1985;312:665-673.

2. Fisher B, Redmond C, Poisson R, et al. Eight-year results of a randomised clinical trial comparing total mastectomy and lumpectomy with or without irradiation in the treatment of breast cancer. N Engl J Med 1994;320:822-828.

3. Jacobson JA, Danforth DN, Cowan KH, et al. Ten-year results of a comparison of conservation with mastectomy in the treatment of stage I and II breast cancer. N Engl J Med 1995;332: 907-911.

4. Ismail J, Proschan MA. Randomised trials of breast-conserving therapy versus mastectomy for primary breast cancer: a pooled analysis of updated results. Am J Clin Oncol 2005;28:289-294.

5. Lee-Feldstein A, Anton-Culver H, Feldstein PJ. Treatment differences and other prognostic factors related to breast cancer survival. Delivery systems and medical outcomes. JAMA 1994; 271:1163-1168.

6. Winchester DJ, Menck HR, Winchester DP. The National Cancer Database report on the results of a large nonrandomised comparison of breast preservation and modified radical mastectomy. Cancer 1997;80:162-167.

7. Cohen L, Hack TF, de Moor C, et al. The effects of type of surgery and time on psychological adjustment in women after breast cancer treatment. Ann Surg Oncol 2000;7:424-434.

8. Martin MA, Meyricke R, O’Neill T, Roberts S. Factors affecting hospital readmission rates for breast cancer patients in Western Australia. Under review.

9. Giles GG. Medical record linkage in Australia. This is as good as it gets. ANZ J Surg 2005;75:259.

10. Foo CS, Su D, Chong CK, et al. Breast cancer in young Asian women: study on survival. ANZ J Surg 2005;75:566-572.

11. Jayasinghe UW, Taylor R, Boyages J. Is age at diagnosis an independent prognostic factor for survival following breast cancer? ANZ J Surg 2005;75:762-767.

12. Prentice RL, Williams BJ, Peterson AV. On the regression analysis of multivariate failure time data. Biometrika 1981;68:373-379.

13. Therneau TM, Grambsch PM. Modelling survival data: extending the Cox model. New York: Springer-Verlag; 2000.

14. Taylor KO. Morbidity associate with axillary surgery for breast cancer. ANZ J Surg 2004;74:314-317.

15. Shrank P, Rieger R, Shamiyeh A, Wayand W. Morbidity following sentinel lymph node biopsy versus axillary lymph node dissection for patients with breast carcinoma. Cancer 2000;83:608-614.

16. Hynes D, Weaver F, Morrow M, et al. Breast cancer surgery trends and outcomes: Results from a national Department of Veterans Affairs study. J Am Coll Surg 2004;198:707-716.

17. National Health and Medical Research Council. Clinical practice guidelines for the management of early breast cancer. 2nd ed. Canberra: National Health and Medical Research Council; 2001.

18. National Comprehensive Cancer Network. Breast cancer: clinical practice guidelines in oncology, v. 2.2006. Available at: http://www.nccn.org/professionals/physician_gls/default.asp 